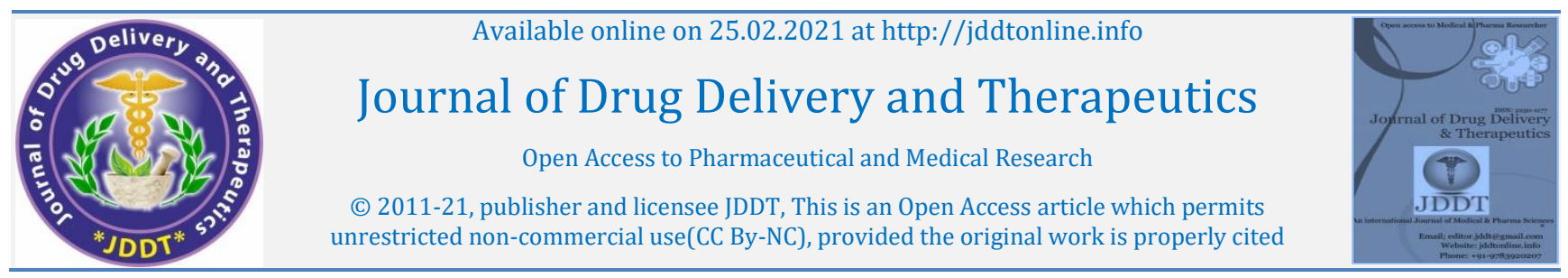

(c) 2011-21, publisher and licensee JDDT, This is an Open Access article which permits Open Access Full Text Article

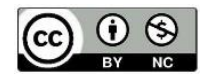

Research Article

\title{
Evaluation of Efficacy of Cucumis melo in Gentamycin and CPD Induced Urolithiasis on Rats
}

\author{
Patel Dhaval $\mathrm{M}^{1 *}$, Patel Advaita B ${ }^{1}$, Patel Bhumi R ${ }^{1}$, Patel Deepa R ${ }^{2}$, Patel Nishith $\mathrm{K}^{3}$ \\ ${ }^{1}$ SAL Institute of Pharmacy, Opp-Science city, Ahmedabad-380060, Gujarat, India
}

${ }^{2}$ Kalol Institute of Pharmacy, Kalol, Gandhinagar, Gujarat, India

${ }^{3}$ Arihant School of Pharmacy and Bio-Research Institute, Gandhinagar, Gujarat, India

Article Info:
Article History:
$\begin{aligned} & \text { Received } 02 \text { Jan 2021; } \\ & \text { Review Completed 10 Feb } 2021 \\ & \text { Accepted } 19 \text { Feb 2021; } \\ & \text { Available online 25 Feb } 2021\end{aligned}$
$\begin{aligned} & \text { Patel DM, Patel AB, Patel BR, Patel DR, Patel NK, } \\ & \text { Evaluation of Efficacy of Cucumis melo in Gentamycin } \\ & \text { and CPD Induced Urolithiasis on Rats, Journal of Drug } \\ & \text { Delivery and Therapeutics. 2021; 11(1-s):113-116 } \\ & \text { DOI: http://dx.doi.org/10.22270/jddt.v11i1-s.4568 }\end{aligned}$

*Address for Correspondence:

Patel Dhaval M, SAL Institute of Pharmacy, Opp-Science city, Ahmedabad-380060, Gujarat, India

\author{
Abstract
}

Evaluation of the efficacy of methanolic extract of Cucumis melo in urolithiasis induced by gentamycin and calculi producing diet on Wistar rats. Gentamycin $(40 \mathrm{mg} / \mathrm{kg}$, subcutaneously) and calculi-producing diet (CPD) was fed to induce urolithiasis on Wistar rats. The effect of oral administration of methanolic extract of Cucumis melo seed on calcium oxalate urolithiasis has been studied and is compared with the effect of oral administration of Cystone as standard on Wistar rats. Gentamycin and CPD feeding resulted in hyperoxaluria and calcium oxalate deposition as well as increased renal excretion of calcium and oxalate. Supplementation with methanolic extract of Cucumis melo seed reduced the elevated urinary oxalate, showing a regulatory action on endogenous oxalate synthesis. The results indicate that the seed of Cucumis melo is endowed with antiurolithiatic activity.

Keywords: C. melo, Hyperoxaluria, calcium oxalate deposition, cystone, hyperoxaluria, analysis of variance

\section{INTRODUCTION}

Urolithiasis means the process of forming calculi (stones) in the urinary tract (kidney, urinary bladder, and/or urethra). A renal calculus is a hard mineral and crystalline material formed within the kidney or urinary tract. Kidney calculi are a common cause of blood in the urine and often severe pain in the abdomen, groin or flank. Kidney calculi are sometimes called renal calculi. 5\% people develop a kidney calculus at some point in their life. The development of the calculi is related to reduce urine volume or increased excretion of calculus-forming components such as calcium, oxalate, urate, cystine, xanthine and phosphate. Kidney calculi are composed of organic and inorganic crystals amalgamated with proteins. Crystallization and subsequent lithogenesis can happen with many solutes in the urine. Calcareous calculi are still by far the most common nephroliths, accounting for more than $80 \%$ of calculi. ${ }^{1-6}$

The prevalence and incidence are estimated to be $5-10 \%$ and $100-300 / 100,000 /$ year, respectively. Relapses occur in 50$70 \%$ of all cases. ${ }^{7}$ At present the prevalence occurs in ratio of $6 \%$ in women and $15 \%$ in men. This increase is linked to changes in lifestyle, eating patterns and obesity. The complications of urolithiasis are obstruction and uremia, sepsis, chronic pyelonephritis, acute or chronic renal failure, xanthogranulomatous pyelonephritis, emphysematous pyelonephritis (EPN), pyonephrosis, hypertension, etc. The ISSN: 2250-1177 [113] mortality/morbidity of urolithiasis depends on (a) Acute ureteral obstruction by calculus causes severe, colicky (intermittent) flank pain that can radiate throughout the groin, testicles, back, or periumbilical region. Some patients with renal calculi may have no symptoms at all. Calculi smaller than $4 \mathrm{~mm}$ pass spontaneously in approximately $80 \%$ of patients. Calculi that are $4-6 \mathrm{~mm}$ pass in approximately $50 \%$ of patients, whereas calculi larger than 8 $\mathrm{mm}$ pass in only approximately $20 \%$ of patients. (b) Occasionally, recurrent infection may result in pyelonephritis or abscess. Calculi can cause renal scarring, damage, or even renal failure if they are bilateral. In $10 \%$ of patients, calculi reoccur within 1 year. This percentage increases to $50 \%$ within 10 years. (c) Passage of a renal calculus is the most common cause of acute ureteral obstruction. When this occurs, pressure in the collecting system and renal blood flow acutely increases, followed by decreased blood flow after 1-2 hours. Hematuria usually occurs. This can be intermittent or persistent and microscopic or gross. However, as many as $10 \%$ of patients with acute calculi may not have hematuria. 8-10

Currently treatment options for nephrolithiasis are very limited. For smaller calculus, advices to drink more fluid \& pain medications including Non-Steroidal Anti-inflammatory Drugs (NSAIDs) are suggested. To enhance passage of calculus from urinary tract, diuretics are prescribed. Flush 
therapy is also suggested for that. For larger \&/or painful calculi, lithotripsy \& some other surgical procedures are preferred.

Diuretics can cause severe adverse effects such as ototoxicity, acute hypovolemia, potassium depletion, hyponatremia. Pain Medication like NSAIDs causes number of side effects like peptic ulcer, nephrotoxicity etc. Surgical procedure has its own sets of limitation like risk for postoperative complication, cost, specific skill \& equipments requirement. So, many herbal drugs are used in treatment of urolithiasis. Mostly used herbal drugs are Tribulus terrestris, Crataeva nurvala, Bergenia ligulata, Hemidesmus indicus, Foeniculum vulgare etc. Cassia occidentalis is used as antiasthmatic activity. ${ }^{11-16}$

Allopath does not have any specific medication for Urolithiasis. Options available today have many limitations including risk of adverse effects, where as herbal world offers many options with safety, efficacy \& availability with economical aspects too. Cucumis melo, commonly known as "Musk melon", is one of the leading drugs used as alternative system of treatments. As per traditional method, it is known for its diuretic activity.

\section{MATERIALS AND METHODS}

\section{Plant Material}

A seed powder of Cucumis melo was procured from L. V. Gandhi stores at Gandhi bridge, Ahmedabad. The seed powder of Cucumis melo was subjected to extraction using soxhlet apparatus. The powdered material was exhaustively extracted with methanol in soxhlet apparatus by continuous hot extraction. After each extraction, the solvent was recovered using distillation assembly, and the extract was concentrated under reduced pressure. The final yield of extract was stored in air tied container for experiment.

\section{Animal selection}

Healthy adult Wistar Albino rats (200-400gm) were selected for study of antiurolithiatic activity. The animals were acclimatized to standard laboratory conditions (temperature: $25 \pm 5{ }^{\circ} \mathrm{C}$ ), humidity $(55 \pm 5 \%)$ and maintained on 12-h light: 12-h dark cycle. They were provided with regular rat chow and drinking water ad libitum. The experimental protocol was approved by Institutional Animal Ethics Committee as per the guidance of committee for the purpose of Control and Supervision of Experiments on Animals (CPCSEA), Ministry of Social Justice and Empowerment, Government of India.

\section{Gentamycin and CPD induced urolithiasis model 17}

Hyperoxaluria and calcium oxalate deposition was induced by gentamycin $(40 \mathrm{mg} / \mathrm{kg}$, subcutaneously) and calculiproducing diet (CPD). The standard rat pellet feed was powdered and mixed with ammonium oxalate $(5 \%)$ and gain made into pellets. This feed is called a calculi producing diet and it was fed to animals to induce hyperoxaluria and calcium oxalate calculi. The animals were divided into four groups containing six animals in each. The animals received seed extract or vehicle orally. Except group I all animals received calculi-producing diet and drinking water ad libitum throughout study period. Calculi-producing diet was given for 10 days (5-15th day). And group I received regular rat food and drinking water ad libitum. Gentamycin (40 $\mathrm{mg} / \mathrm{kg}$ ) is administered subcutaneously for 8 days. Group III received standard antiurolithiatic drug, cystone $(750 \mathrm{mg} / \mathrm{kg}$ body weight) once daily p.o. upto 30 th day. While group IV received Cucumis melo (300 mg/kg body weight) orally once daily throughout study period.

\section{Assessment of antiurolithiatic activity}

Collection and analysis of urine: The rats were hydrated with $5 \mathrm{ml}$ of distilled water orally, placed in separate metabolic cages, and urine samples were collected for $24 \mathrm{~h}$ from all groups at the end of the experimental period (day 30). The samples were centrifuged at 2,500 rpm for $5 \mathrm{~min}$. The supernatant was used to estimate calcium and oxalate. ${ }^{18-19}$

Kidney homogenate analysis: At the end of the study, animals were decapitated, kidneys were perfused with ice-cold saline $(0.9 \% \mathrm{w} / \mathrm{v}$ sodium chloride), and one kidney from each animal was carefully incised, washed in ice-cold $0.15 \mathrm{M} \mathrm{KCl}$ and was homogenized in $10 \% \mathrm{HCl}$. The homogenate was centrifuged at 2,500 rpm for $3 \mathrm{~min}$ and the supernatant was used to estimate calcium and oxalate 20-23.

\section{Statistical analysis}

Results were expressed as mean \pm SD. Differences among data were determined using one-way ANOVA followed by Student-Newman-Keul's test (Graphpad Prism software for Windows, Version 4.10.1998). Differences between the data were considered significant at $P<0.05$.

\section{RESULTS \& DISCUSSION}

\section{Result}

In the present study, chronic administration of gentamycin ( $40 \mathrm{mg} / \mathrm{kg}$ body weight) and calculi producing diet to male Wistar rats resulted in hyperoxaluria.

Urine calcium level was significantly elevated in model group $(14.46 \pm 0.527 \mathrm{mg} / \mathrm{dl} ; \mathrm{p} \leq 0.001)$ as compared to normal animals $(9.36 \pm 0.555 \mathrm{mg} / \mathrm{dl})$. This increase was significantly lowered by treatment with cystone $(8.83 \pm 0.724 \mathrm{mg} / \mathrm{dl} ; \mathrm{p} \leq$ $0.001)$, C. melo $(8.57 \pm 0.694 \mathrm{mg} / \mathrm{dl} ; \mathrm{p} \leq 0.001)$ groups as compared to model. There was significant increase in calcium in kidney in model group $(8.51 \pm 0.260 \mathrm{mg} / \mathrm{dl}$; $\mathrm{p} \leq$ $0.001)$ as compared to normal control group (3.88 \pm 0.325 $\mathrm{mg} / \mathrm{dl})$. Significant reduction was observed in cystone $(4.36 \pm 0.260 \mathrm{mg} / \mathrm{dl} ; \mathrm{p} \leq 0.001)$ and C. melo $(4.27 \pm 0.305$ $\mathrm{mg} / \mathrm{dl} ; \mathrm{p} \leq 0.001$ ) treated groups as compared to model group. There was no significant decrease in urine oxalate level in model group as compared to normal control group. There was significantly increase in oxalate level in kidney in model animals $(0.71 \pm 0.080 \mathrm{mg} / \mathrm{dl} ; \mathrm{p} \leq 0.001)$ as compared to normal control animals $(0.31 \pm 0.031 \mathrm{mg} / \mathrm{dl}$; $\mathrm{p} \leq 0.001)$. Additionally significantly reduction in oxalate level was also observed in cystone $(0.37 \pm 0.040 \mathrm{mg} / \mathrm{dl} ; \mathrm{p} \leq 0.001)$ and $C$. melo $(0.34 \pm 0.050 \mathrm{mg} / \mathrm{dl} ; \mathrm{p} \leq 0.001)$ treated groups as compared to model. (Table 1). 
Table 1 Effect of $C$. melo seed extracts on urinary parameters in control and experimental animals (mean \pm SD)

\begin{tabular}{|l|l|l|l|l|}
\hline Parameter (unit) & Group I control & Group II calculi induced & Group II cystone treated & Group IV C. melo treated \\
\hline Urine (mg/ml) & \multicolumn{5}{|l|}{} \\
\hline Oxalate & $0.36 \pm 0.150$ & $0.76 \pm 0.150$ & $0.43 \pm 0.120$ & $0.37 \pm 0.160$ \\
\hline Calcium & $9.36 \pm 0.555$ & $14.46 \pm 0.527$ & $8.83 \pm 0.724$ & $8.57 \pm 0.694$ \\
\hline
\end{tabular}

Table 2 Effect of $C$. melo seed extracts on kidney parameters in control and experimental animals (mean \pm SD)

\begin{tabular}{|l|l|l|l|l|}
\hline Parameter (unit) & Group I control & Group II calculi induced & Group II cystone treated & Group IV C. melo treated \\
\hline Kidney (mg) & $0.31 \pm 0.031$ & $0.71 \pm 0.080$ & $0.37 \pm 0.040$ & $0.34 \pm 0.050$ \\
\hline Oxalate & $3.88 \pm 0.325$ & $8.51 \pm 0.260$ & $4.36 \pm 0.260$ & $4.27 \pm 0.305$ \\
\hline Calcium &
\end{tabular}

\section{DISCUSSION}

Focal necrosis, the loss of border membranes and the occurrence of membrane debris in the tubule lamina are a few of the factors that induce renal calculus formation. ${ }^{24}$ Treatment with high doses of gentamycin in rats was reported to induce calculus formation by causing such damage. The membranous debris produced by gentamycin acts as a nucleation site for calcium oxalate crystallization. Moreover, gentamycin has been shown to inhibit calcium reabsorption in proximal tubules. ${ }^{25}$ Hyperoxaluria induces not only calcium oxalate crystallization but also papillary damage. ${ }^{26}$ A similar elevation of renal calculus-forming constituents in rats fed with calculi-producing diet using glycolic acid was reported previously.27 $0 n$ the administration of gentamicin and CPD an increase in the deposition of calcium and oxalate was observed. Administration of extract of C. melo given significantly reduced both calcium and oxalate deposition in the kidneys of drug treated groups when compared to model controls. Urinary supersaturation with respect to calculus-forming constituents is one of the causative factors in calculogenesis. In this context, the changes in the urinary oxalate levels are relatively much more important than those of calcium. ${ }^{28}$ It has been reported that oxalate plays an important role in calculus formation and has about a 15-fold greater effect than urinary calcium. ${ }^{29}$ Oxalate salts are soluble when formed with magnesium but when complexed with calcium they form insoluble calcium oxalate, causing crystalline precipitation of renal calcium oxalate calculi. ${ }^{30-31}$ In the present study, the ability of C. melo to alter calcium and oxalate excretion and deposition may be due to the disintegration of mucoproteins, which are actually promoters of crystallization. A similar mechanism for antiurolithiatic agents was reported previously by Grases et al. ${ }^{32}$ Some herbal remedies have antimicrobial properties that protect the anti-adherent layer of the mucosa which acts as a protective barrier against urinary calculi.

This may also be responsible for the significant antiurolithiatic activity of C. melo, as earlier reports suggested that $C$. melo had antimicrobial properties against several skin and gastrointestinal tract-infecting microbes. ${ }^{33}$ The presence of polyphenols might help in dissolving calcium oxalate and magnesium ammonium phosphate calculi by forming complexes with divalent cations such as calcium and magnesium. ${ }^{34}$ This complexation may reduce the availability of calcium for absorption, thus reducing both the excretion and the deposition of calcium in kidney.

\section{CONCLUSION}

The presented data indicate that administration of the methanolic extract of Cucumis melo seed to rats with gentamycin and CPD induced lithiasis, reduced and prevented the growth of urinary calculi, supporting folk information regarding antiurolithiatic activity of the plant. These effects could conclude that Cucumis melo has an antiurolithiatic property.

\section{REFERENCES}

1. Heller H., Sakhaee K., Moe O.W., Pak C.Y. Etiological role of estrogen status on renal calculus formation. J. Urol., 2002; 168:1923-1927.

2. Harvey J.A., Hill K.D., Pak C.Y. Similarity of urinary risk factors among calculus-forming patients in five regions of the United States. J. Lithotr. Calculus Dis., 1990; 2:124-132.

3. Herring L.C. Observations on the analysis of ten thousand urinary calculi. J. Urol., 1962; 88:545-562.

4. Daudon M., Donsimoni R., Hennequin C., Lacour B., Moel G., Donsimoni R. Sex- and age-related composition of 10617 calculi analyzed by infrared spectroscopy. Urol. Res., 2005; 23:319-326.

5. Mandel N.S., Mandel G.S. Urinary tract calculus disease in the United States veterans population. I. Geographic frequency of occurrence. J. Urol., 1989; 142:1513-1515.

6. Patel D.M., Patel A.B., Trivedi R.D., Parmar V.J., Bangoriya U.V Evaluation of the effect of hydroalcoholic extract of Cassia occidentalis leaves in neutrophil adhesion test in rats. Journal of Drug Delivery and Therapeutics. 2019; 9(4s):1218-1221. DOI 10.22270/jddt.v9i4-s.3940

7. Saita A., Bonaccorsi A., Motta M. Calculus composition: where do we stand? Urol. Int. 2007; 79(1):16-19.

8. Al-Mamari S.A. Complications of urolithiasis, Urolithiasis in Clinical Practice. Springer Publication, 2017; 121-129.

9. Patel A., Patel D., Patel D., Sheth A., Shah U.J. Method Development and Validation for Simultaneous Estimation of Benidipine Hydrochloride and Metoprolol Succinate in Tablet. Journal of Drug Delivery and Therapeutics. 2019; 9(6-s):28-33. DOI 10.22270/jddt.v9i6-s.3692

10. Patel D.M., Trivedi R., Patel H. Formulation and Evaluation of BiLayer Tablets of Ketorolac Tromethamine. Journal of Drug Delivery and Therapeutics. 2021; 11(1):36-41. DOI 10.22270/jddt.v11i1.4487

11. Bangoriya U.V., Tank H.M., Patel D.M., Tank P.H. Evaluation of the effect of hydroalcoholic extracts of Cassia occidentalis in clonidine induced mast cell degradation in Rats. JPSBR. 2016; 6 (1):120-123.

12. Patel D.M., Tank H.M., Bangoriya U.V., Tank P.H. Evaluation of the effect of hydroalcoholic extracts of Cassia occidentalis in carrageenan induced paw edema in Rats. JPSBR. 2016; 6 (1):157-161.

13. Bangoriya U.V., Tank H.M., Patel D.M., Tank P.H. Evaluation of the effect of hydroalcoholic extracts of Cassia occidentalis in carrageenan induced paw edema in rats. Pharma Science 
Monitor: An International Journal Of Pharmaceutical Sciences. 2015; 6 (3):65-72.

14. Bangoriya U.V., Tank H.M., Patel D.M., Tank P.H. Evaluation of the effect of hydroalcoholic extracts of Cassia occidentalis in histamine induced bronchospasm on guinea pigs. Pharma Science Monitor: An International Journal of Pharmaceutical Sciences. 2015; 6 (4):118-124.

15. Patel D.M., Bangoriya U.V., Tank H.M., Tank P.H. Evaluation of the effect of hydroalcoholic extracts of Cassia occidentalis seeds in clonidine induced mast cell degradation in Rats. JPSBR. 2017; 7 (5):346-349.

16. Patel D.M., Patel A.B., Trivedi R.D., Parmar V.J., Bangoriya U.V. Evaluation of the effect of hydroalcoholic extracts of Cassia occidentalis leaves in neutrophil adhesion test in rats. Journal of Drug Delivery and Therapeutics. 2019; 9(4s):1218-1221. DOI 10.22270/jddt.v9i4-s.3940

17. Patel D.M., Lumbhani A.N. Evaluation of the efficacy of methanolic extract of Foeniculum vulgare in urolithiasis on Wistar rats. Int J Pharmacy Res and Tech, 2013; 3(2):12-15.

18. Medeiros D.M., Mustafa M.A. Proximate composition, mineral content and fatty acids of cat fish (Ictalurus punctatus rafinesque) for different seasons and cooking methods. J. Food Sci., 1985; 50:585-588

19. Hodgkinson A., Williams, A. An improved colorimetric procedure for urine oxalate. Clinica Chimica Acta, 1972; 36:127-132.

20. Patel A.B., Patel D.R., Patel D.M., Patel K.S., Patel P. Stability indicating HPLC method development and validation of Enrofloxacin and Bromhexine $\mathrm{HCl}$ in combined dosage form. Aegaeum Journal. 2020; 8 (5):443-457.

21. Khan S.R., Hackett R.L. Membrane induced calcium oxalate crystal nucleation. Urol Res, 1988; 16:185.

22. Nakarani P.P., Patel, D.M. Development and Validation of Stability Indicating HPTLC Method for Estimation of Acebrophylline in Their Dosage Form. IJPRT. 2013; 3 (1):1-4.

23. Patel D.M., Patel D., Patel A., Sheth A., Shah U.J. Method Development and Validation for Simultaneous Estimation of Benidipine Hydrochloride and Metoprolol Succinate in Tablet. Journal of Drug Delivery and Therapeutics. 2019; 9(6-s):28-33. DOI 10.22270/jddt.v9i6-s.3692
24. Chahwala S.B., Harpur E.S. Gentamicin-induced hypercalciuria in the rat. Acta Pharmacol Toxicol, 1983; 53:358.

25. Baumann M.J. Calculus prevention: why so little progress? Urol Res, 1998; 26:77-81.

26. Baskar R., Malini M.M., Varalakshmi P., Balakrishna K., Rao, R.B. Effect of Lupeol isolated from Crataeva nurvala stem bark against free radical induced toxicity in experimental urolithiasis. Fitoterapia, 1996; 2:121-125.

27. Robertson W.G., Peacock M. The course of idiopathic calcium disease: hypercalciuria or hyperoxaluaria. Nephron, 1980; 26:105-110.

28. Borghi, L, Meschi T., Guerra A., Novanni A. Randomized prospective study of a nonthiazide diuretic, indapamide, in preventing calcium calculi recurrences. J Cardiovasc Pharmacol, 1993; 22:78-86.

29. Marshall R.W., Robertson W.G., Nonograms for the estimation of the saturation of urine with calcium oxalate, calcium phosphate, magnesium ammonium phosphate, uric acid, sodium acid urate. Clin Chim Acta, 1976; 72:253-257.

30. Pak C.Y.C., A cautious use of cellulose phosphate in the management of calcium nephrolithiasis. Invest Urol, 1981; 19:187-190.

31. Jha A.N., Patel D.M. Role of Cannabinoid receptors in psychological disorder. Borneo Journal of Pharmacology. 2020; 3 (4):199-208.

32. Grases F., March J.G., Ramis M., Costa-Bauza A. The influence of Zea mays on urinary risk factors for kidney calculi in rats. Phytother Res, 1993; 7:146-149.

33. Silva H.J.R.L., Anusari L.B.W., Ratnasooriya W.D., Pathirana R.N., Widanagamage R.D. In vitro antibacterial activity and the minimum inhibitory concentration of aqueous seeds extract of Cucumis melo L. grown in Sri Lanka on common urinary tract infective bacteria. Journal of Pharmacognosy and Phytochemistry, 2018; 7(3):686-690.

34. Grases F., Ramis M., Costa-Bauza A., March J.G. Effect of Herniaria hirsuta and Agropyron repens on calcium oxalate urolithiasis in rats. J Ethnopharmacol, 1995; 45:211-214. 